\title{
Numerical analysis of particle recycling in the TEXTOR helical divertor
}

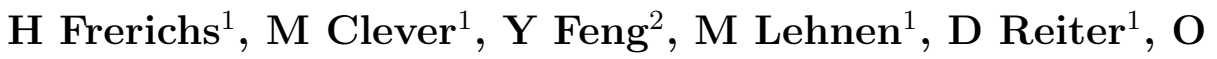 \\ Schmitz ${ }^{1}$ \\ ${ }^{1}$ Institute of Energy and Climate Research - Plasma Physics, Forschungszentrum \\ Jülich GmbH, Association EURATOM-FZJ, Partner in the Trilateral Euregio \\ Cluster, Jülich, Germany \\ 2 Max-Planck Institute for Plasma Physics, 17491 Greifswald, Germany \\ E-mail: h.frerichs@fz-juelich.de
}

\begin{abstract}
The TEXTOR helical divertor is a magnetic configuration created by the application of external resonant magnetic perturbations (RMPs) with the intention to control plasma edge transport and the resulting particle and heat fluxes to the divertor target. It is confirmed by 3D computer simulations that no high-recyclinglike regime is established under TEXTOR relevant conditions, despite the fact that a transition to detachment (i.e. a saturation or even a roll-over of the recycling flux) is observed at high densities. The driving mechanisms are, distinct from apparently similar observations in poloidal divertors and stellarator divertors, a combination of volumetric power losses and enhanced upstream-to-downstream heat transport, but with no significant role of the momentum balance.

PACS numbers: 52.25.Fi, 52.55.Rk, 52.65.-y, 52.65.Kj
\end{abstract}

Submitted to: Nucl. Fusion

\section{Introduction}

External resonant magnetic perturbations (RMPs) are applied in magnetically confining toroidal fusion devices with the intention to control plasma edge transport and the resulting particle and heat fluxes to the divertor target. The TEXTOR helical divertor is one such configuration, which continues earlier RMP related research, e.g. at Tore Supra with its ergodic divertor $[1,2,3,4,5]$. As a result of RMP application at TEXTOR, a 3D open chaotic magnetic field structure with helical strike lines is generated at the plasma edge $[6,7,8]$, similar to the magnetic structure of divertors in stellarators such as LHD $[9,10]$. This perturbed helical structure allows to address basic transport mechanisms relevant for both magnetic confinement machine types: tokamaks and stellarators.

A significant impact of this magnetic structure on plasma transport has been observed experimentally $[11,12,13,14,15]$. However, the modification of edge plasma 


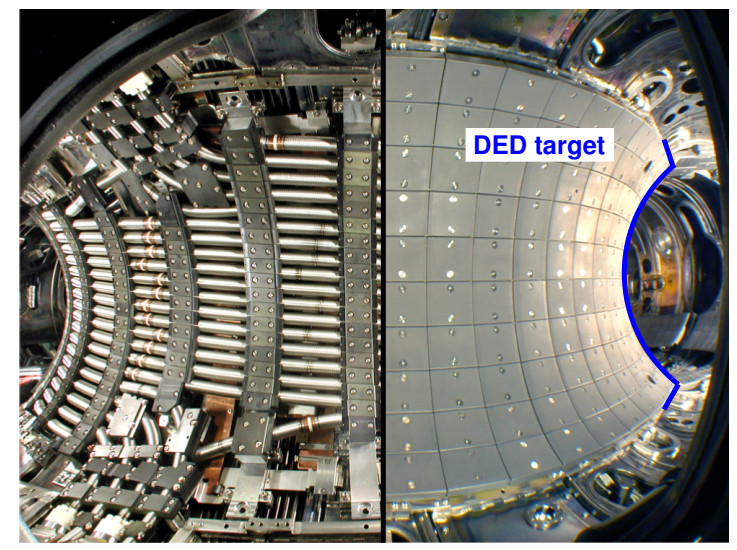

Figure 1. Setup of the helical coil system of the DED at TEXTOR. The coils are covered by graphite plates (the DED target) for protection during plasma discharges.

parameters - and consequently of particle and heat fluxes to the divertor target - are still in the focus of ongoing research, in particular since RMPs have been found to be a promising tool to control edge localized modes (ELMs) in DIII-D [16] and JET [17]. Detailed understanding of the impact of RMPs on the edge plasma characteristics may therefore be an important step towards a reliable and trustworthy design of the ITER ELM mitigation coil system. ELM mitigation as such is a critical issue, because otherwise the ELM induced high transient particle and heat loads to the first wall and divertor target are predicted to significantly reduce the ITER wall life-time [18, 19]. Furthermore, the perturbed magnetic configuration at TEXTOR is general enough to be relevant for stellarator research as well, because such 3D effects are intrinsically present in these devices.

The aim of the present paper is to study the particle recycling characteristics of the TEXTOR helical divertor configuration. Experimental observations [20, 21] indicate a deviation from the common (i.e. poloidal) divertor phases from low and high recycling up to detachment, which is similar to observations at W7-AS [22], but unlike those at the Tore Supra ergodic divertor which exhibits all three regimes [1, 2, 23, 24]. The so called dynamic ergodic divertor (DED), a helical coil set located at the high field side (HFS) at TEXTOR (see figure 1), is a flexible tool to generate a spectrum of RMPs with main resonances around the $q=3$ surface (which is located at the plasma edge in the discharges considered here). Poloidal/toroidal base mode numbers $m / n=3 / 1$, as well as $6 / 2$ and $12 / 4$ can be selected by a different wiring of the perturbation coils. In the present analysis we focus on the $m / n=6 / 2$ configuration in DC operation, which is described in section 2 in more detail.

The present studies have been performed with the 3D transport code package EMC3-EIRENE, which is a coupled version of the 3D fluid edge plasma transport code EMC3 [25, 26] and the 3D kinetic neutral particle transport code EIRENE [27]. An outline of the model equations solved by the code is given in section 3 . This code has 
been adapted to conditions at TEXTOR [28] and has already been used for simulations of configurations with base mode number 12/4 [29].

Divertor properties are often analysed within so called 2-point models [30], in which upstream and downstream parameters in the scrape-off layer (SOL) are related to target particle and heat fluxes. These models can give insight into the general operational regime, but naturally suffer from varios geometry and transport related simplifications. Extensions of the basic 2-point model have been proposed in [30] as well, including the general effects of momentum losses, contributions from convective heat transport and volumetric power losses as external model parameters. In [31] an improved 2-point model for the TEXTOR limiter edge has been presented, with parallel convective transport included in a self-consistent way, but with simplified description of the recycling zone and neutral particle transport. Here the importance of convective transport and volumetric losses due to interactions with neutrals for the deviation from the strong recycling regime at a high density were demonstrated. There, despite the strong simplifications made, a striking good (even quantitative) agreement with 2D (toroidally symmetric) full calculations by the EMC3-EIRENE code package was found. This indicates that the collisionality dependence of the ratio of electron temperatures at the mid-plane and near the limiter differs significantly from that prescribed by the standard 2-point model, if one assumes strong recycling at the limiter/divertor targets.

A generalization of these models for a 3D chaotic SOL with helical divertor strike lines is challenging, if possible at all, already because no obvious definition of the upstream position exists. In this case 3D computer simulations provide a valuable tool for the quantification of the RMP induced effects on the plasma edge. Simulation results of the total (i.e. target surface integrated) and local recycling flux scaling with density are presented in section 4 . Then, in section 5, we give an interpretation of the simulation result which is guided by the ad hoc correction factors introduced in the extended 2-point model in [30]. A similar approach was applied in [32] for the interpretation of 2D numerical results from the B2-EIRENE code modeling. However, in [32] a special form of 2-point equations, which is exact in the frame of the code model, was introduced. Contrary to that, the analysis in the present work is based on the standard approximate 2-point relations which are used to estimate the relationship

between underlying transport processes in order to separate the most important driving mechanisms.

\section{The helical divertor configuration at TEXTOR}

The transport studies presented below are based on the MHD equilibrium of TEXTOR discharge \#95896 with superimposed perturbation field in $m / n=6 / 2$ configuration, powerd by a current of $I_{\mathrm{DED}}=6.75 \mathrm{kA}$. The MHD equilibrium is characterized by a plasma current of $I_{p}=350 \mathrm{kA}$ and a toroidal magnetic field of $B_{\text {tor }}=1.85 \mathrm{~T}$ at the magnetic axis. The DED coils are covered by graphite plates for protection during plasma discharges (see figure 1). These plates are the DED target, which is located 

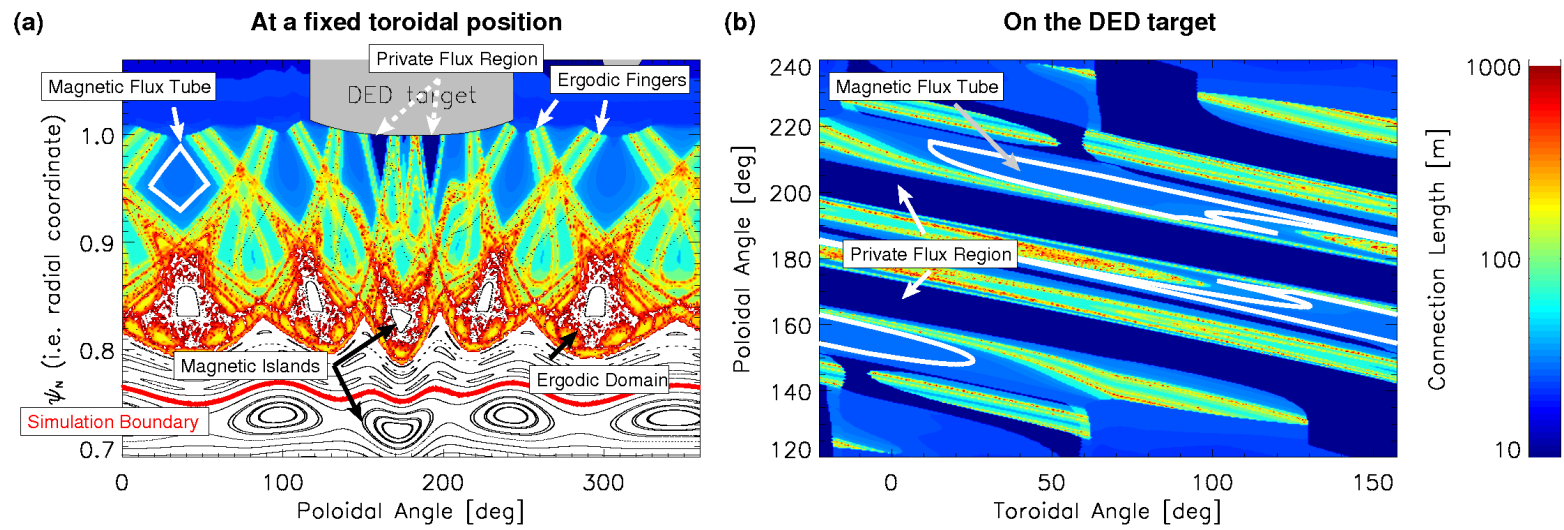

Figure 2. Magnetic field structure of the TEXTOR helical divertor configuration depicted by the wall-to-wall connection length $L_{\mathrm{c}}$. (a) Cut at toroidal position $\varphi=0$ deg with normalized poloidal flux $\Psi_{n}$ as radial coordinate and (b) footprint on the DED target (located at $r_{\min }=47.7 \mathrm{~cm}$ ). Only $\Delta \varphi=180 \mathrm{deg}$ are considered because of the $n=2$ toroidal symmetry.

at a radial position with minor radius $r_{\min }=47.7 \mathrm{~cm}$ and covers the complete toroidal circumference and a poloidal range of $\Delta \vartheta=120 \mathrm{deg}$.

The application of this perturbation field results in the formation of a heterogeneous magnetic field structure at the plasma edge with magnetic island chains, short magnetic flux tubes and chaotic domains with field lines in a wide range of wall-to-wall connection lengths $L_{\mathrm{c}}$ (also referred to as ergodic or stochastic domain). This structure is shown in figure 2 (a) by a $2 \mathrm{D}$ cut at a selected toroidal position (toroidal angle $\varphi=0 \mathrm{deg}$ ) and (b) by a footprint on the DED target. These figures are obtained with field line tracing using a superposition of the equilibrium tokamak field with the perturbation field in vacuum approximation. The contour of a magnetic flux tube and its footprints on the DED target (in positive and negative direction) are marked by white frames. Our focus is on the plasma edge, therefore we use the poloidal angle and normalized poloidal flux as poloidal and radial coordinate, respectively.

\section{Edge transport model}

We now consider plasma (and neutral gas) transport ontop of this prescribed magnetic field structure. Following the approach used in 2D transport modeling, we apply a fluid model for edge plasma transport, but now in 3D (EMC3), which is coupled to a kinetic model for the transport of neutral particles (EIRENE). The EMC3 code solves a set of steady state fluid equations in a 3D space of essentially arbitrary magnetic field geometry [26]:

\section{Plasma density $n$}

$\nabla \cdot\left[n u_{\|} \mathbf{e}_{\|}-D_{\perp} \underline{\mathbf{I}}_{\perp} \cdot \nabla n\right]=S_{p}$ 


\section{Parallel flow $u_{\|}$}

$$
\begin{aligned}
\nabla \cdot \mathbf{e}_{\|}\left[m_{i} n u_{\|}^{2}\right. & \left.-\eta_{\|} \mathbf{e}_{\|} \cdot \nabla u_{\|}\right] \\
& -\nabla \cdot \underline{\mathbf{I}}_{\perp} \cdot D_{\perp} \nabla\left(m_{i} n u_{\|}\right)=-\mathbf{e}_{\|} \cdot \nabla p+S_{m}
\end{aligned}
$$

\section{Electron temperature $T_{e}$}

$$
\begin{aligned}
\nabla \cdot \mathbf{e}_{\|}\left[\frac{5}{2} T_{e} n u_{\|}\right. & \left.-\kappa_{e} \mathbf{e}_{\|} \cdot \nabla T_{e}\right] \\
& -\nabla \cdot \underline{\mathbf{I}}_{\perp} \cdot\left[\chi_{e} n \nabla T_{e}+\frac{5}{2} T_{e} D_{\perp} \nabla n\right]=-k\left(T_{e}-T_{i}\right)+S_{e e}\left(+S_{e, \mathrm{cool}}\right)
\end{aligned}
$$

Ion temperature $T_{i}$

$$
\begin{aligned}
\nabla \cdot \mathbf{e}_{\|}\left[\frac{5}{2} T_{i} n u_{\|}\right. & \left.-\kappa_{i} \mathbf{e}_{\|} \cdot \nabla T_{i}\right] \\
& -\nabla \cdot \underline{\mathbf{I}}_{\perp} \cdot\left[\chi_{i} n \nabla T_{i}+\frac{5}{2} T_{i} D_{\perp} \nabla n\right]=+k\left(T_{e}-T_{i}\right)+S_{e i}
\end{aligned}
$$

where $\mathbf{e}_{\|}$is the unit vector in direction of the magnetic field, $\underline{\mathbf{I}}_{\perp}=\underline{\mathbf{I}}-\mathbf{e}_{\|} \mathbf{e}_{\|}$is the "crossfield tensor" given by the unit tensor $\underline{\mathbf{I}}$ and the dyadic product of the field line direction $\mathbf{e}_{\|}$and $p=n\left(T_{e}+T_{i}\right)$ is the static pressure. Furthermore,

$$
k=\frac{3 m_{e}}{m_{i}} \frac{n}{\tau_{e}}
$$

in the ion-electron energy exchange rate $\pm k\left(T_{e}-T_{i}\right)$, where $\tau_{e}$ is the electron collision time. Parallel transport coefficients $\eta_{\|}, \kappa_{e}, \kappa_{i}$ are taken from the classical transport theory by Braginskii [33], while anomalous cross-field transport is taken into account by free model parameters $D_{\perp}, \chi_{e}, \chi_{i}$, and fixed viscosity $\eta_{\perp}=m_{i} n D_{\perp}$ (the latter has already been applied in (2)). The present version of the EMC3 code includes a simplified description of impurity transport (force balance without the inertial term) and the related energy sink $S_{e, \text { cool }}$ due to impurity radiation [26]. Interactions with neutral particles are taken into account by the sources (or sinks) $S_{p}, S_{m}, S_{e e}$ and $S_{e i}$ in the respective equations for particles, parallel momentum and electron and ion energy. These sources (or sinks) are calculated by the EIRENE code. An iterative procedure is applied to obtain a self-consistent solution for all plasma and neutral particle parameters.

In the following $n_{\text {sepx }}$ denotes the density at the inner simulation boundary (ISB) - which is very close to the last closed magnetic flux surface - and is given in units of $\left[10^{19} \mathrm{~m}^{-3}\right]$. The second characteristic parameter applied as boundary condition is the total power input $P_{\text {in }}$ into the plasma edge, which is calculated from the total heating power substracted by the radiated power in the core.

The impact of the magnetic configuration on the plasma edge, as simulated with the EMC3-EIRENE code, is exemplarily visualized in figure 3 for selected input parameters $n_{\text {sepx }}=10^{19} \mathrm{~m}^{-3}$ and $P_{\text {in }}=600 \mathrm{~kW}$. A significant modification of all plasma parameters is observed. While a constant density and temperature is found on closed magnetic flux surfaces in the axisymmetric case (i.e. on $\Psi_{n}=$ const surfaces for $\Psi_{n}<1$ in figures 3 

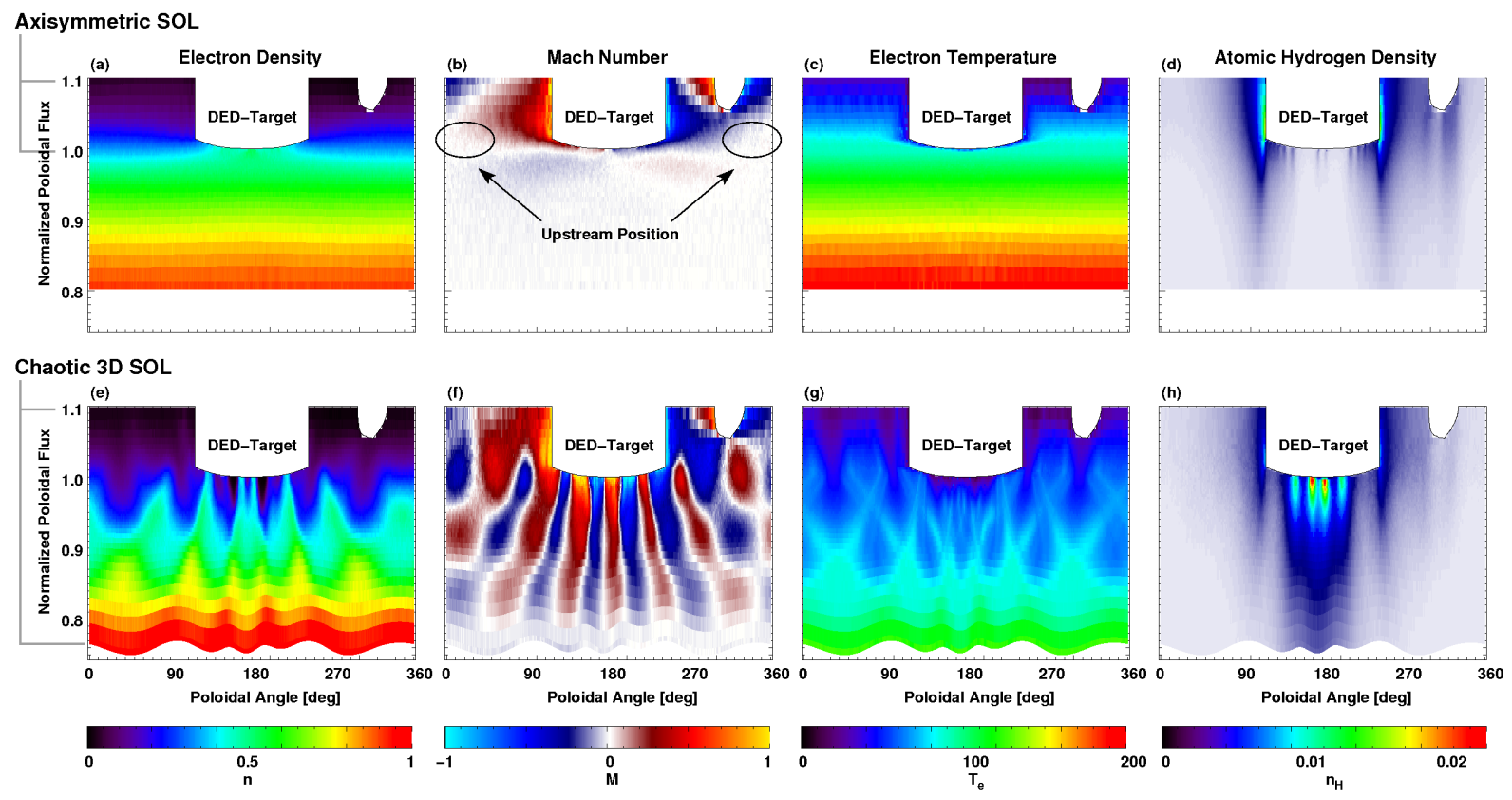

Figure 3. Plasma parameters in the axisymmetric SOL and in the $3 \mathrm{D}$ chaotic SOL, depicted by $2 \mathrm{D}$ cuts at $\varphi=0 \mathrm{deg}$. The electron and atomic hydrogen density is given in units of $\left[10^{19} \mathrm{~m}^{-3}\right]$, the temperature is in $[\mathrm{eV}]$.

(a) and (c)), a strong reduction of both is found in regions with short connection length in the 3D case (in figures $3(\mathrm{e})$ and $(\mathrm{g})$ ). This modification has been analyzed in more detail in [15] and found to be in general agreement with experimental observations.

The main particle flux to the target in the presence of RMPs is diverted from the side surfaces of the DED to the front surface, which is reflected in the distribution of neutral particles (see figure $3(\mathrm{~d})$ and $(\mathrm{h})$ ). It can be seen that neutral particles penetrate deeper into the plasma in terms of $\Psi_{n}$ as radial coordinate, but not with respect to the last closed flux surface (which is pushed inward from $\Psi_{n}=1$ to $\Psi_{n} \approx 0.8$ in the RMP case).

Of particular interest for the following analysis is the development of significant counter-flows in front of the DED target (part (f) in figure 3). Furthermore, the flow pattern in the entire edge domain is modified. Unlike the axisymmetric SOL configuration, in which a well-defined upstream location (i.e. stagnation point) exists, no obvious definition of such a location exists in the 3D chaotic SOL configuration. Therefore, we use the "separatrix density" at the transition between the confined core plasma with closed magnetic flux surfaces and the open chaotic plasma edge region as characteristic parameter for our recycling flux analysis (see red boundary in figure 2 (a)). 


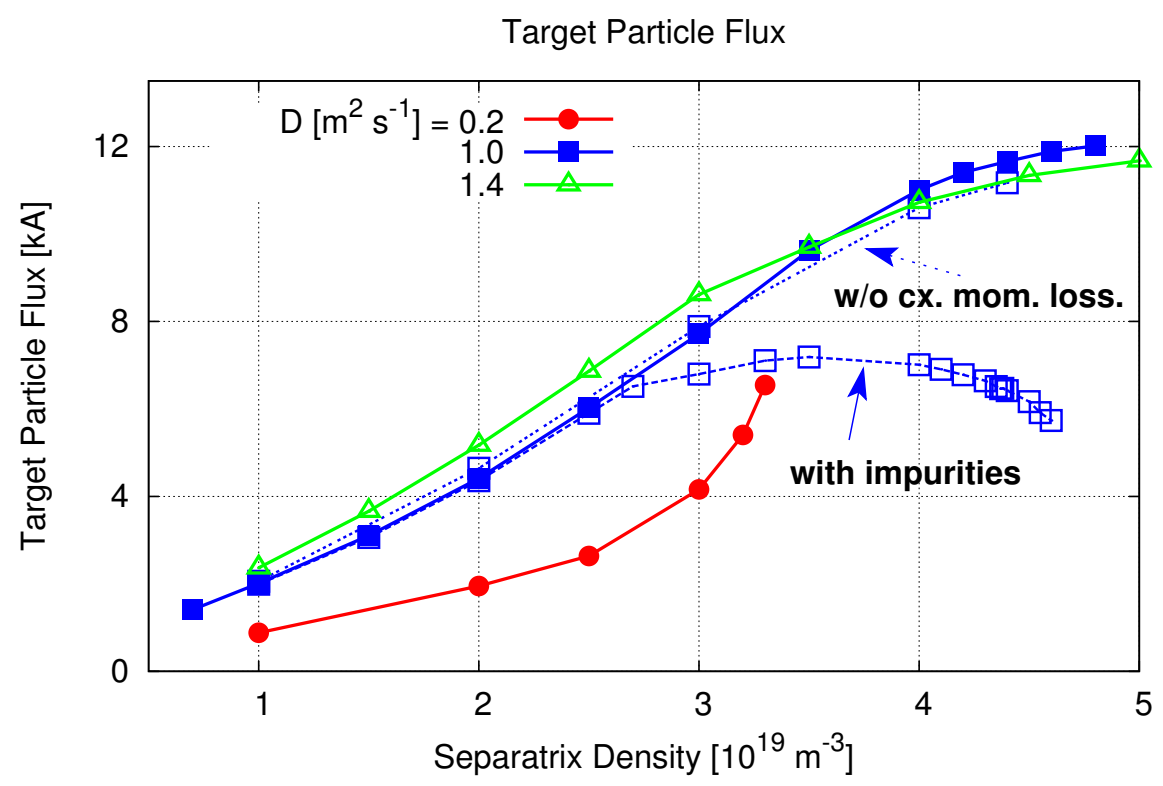

Figure 4. Recycling flux $\Gamma_{t}$ for three levels of anomalous cross-field transport. Additional simulations have been performed in which charge exchange momentum losses have been neglected (blue dotted line) and in which impurities have been included (blue dashed line).

\section{Particle recycling analysis}

Divertor properties are often characterized by the dependence of the so called recycling flux $\Gamma_{t}$ (i.e. the particle flux to the target, which is "recycled" as neutral gas flux) on the upstream density: linear increase in the sheath limited regime at low densities, non-linear increase in the conduction limited regime (high-recycling regime) at higher densities and a "roll-over" into the detachment regime with reduced $\Gamma_{t}$ at even higher densities.

In our modeling analysis, particle recycling in the TEXTOR helical divertor configuration is studied by a scan of the separatrix density $n_{\text {sepx }}$ for three different levels of anomalous cross-field particle diffusion. Anomalous cross-field heat diffusivity is taken to be $\chi_{e}=\chi_{i}=3 D_{\perp}$ throughout all simulations. The input power is set to $P_{\text {in }}=600 \mathrm{~kW}$, which is taken from experimental observations. Impurity production and transport is switched off in the code and will be considered later in section 5.2.2.

\subsection{Characteristics of the total recycling flux}

The total (i.e. integrated over the complete target surface) recycling flux $\Gamma_{t}$ on the DED target is extracted from the EMC3-EIRENE simulations and shown in figure 4. Note that the recycling flux is given by the ion (and electron) current to the target and that we use units of $[\mathrm{A}]$ rather than $\left[\right.$ part. $\mathrm{s}^{-1}$. A non-linear (high-recycling-like) increase of $\Gamma_{t}$ is found only for a low level of anomalous cross-field diffusion $D_{\perp}=0.2$ (values of 


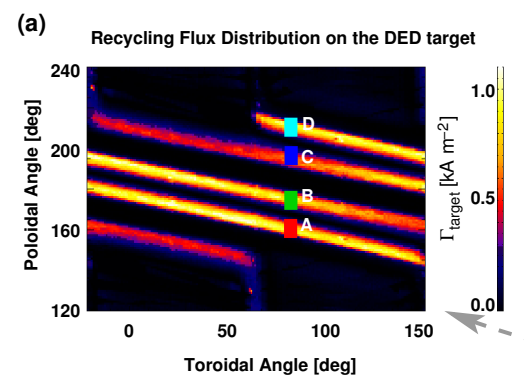

(b)

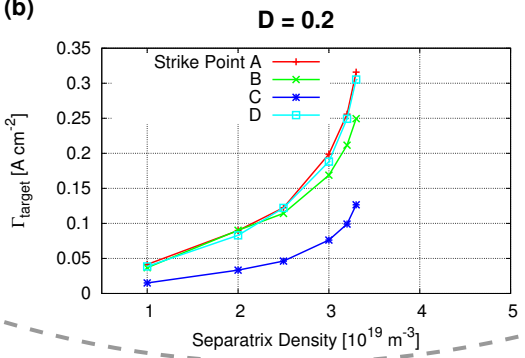

(c)

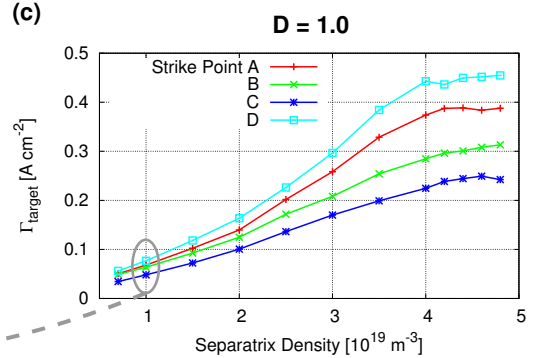

Figure 5. (a) Particle flux distribution for $n_{\mathrm{sepx}}=1.0$ and $D_{\perp}=1.0$. Strike locations A-D are marked by coloured boxes. Local particle flux density at selected strike locations for (b) $D_{\perp}=0.2$ and (c) 1.0 .

$D_{\perp}$ are given in units of $\left[\mathrm{m}^{2} \mathrm{~s}^{-1}\right]$ in the following sections). However, at higher levels of cross-field transport, which are consistent with earlier experimental and modeling results in [15], no high-recycling-like behavior is found prior to saturation of the flux at highest densities. This absense of a high-recycling-like regime is indeed observed in the experiment $[20,21]$, at least in the $m / n=6 / 2$ configuration (in $m / n=3 / 1$ configuration, however, indications for high-recycling are observed [34]). In [35] this behavior is motivated by convective heat fluxes due to a high fraction of upstream sources.

A result of the present simulations for $D_{\perp}=1.0$ and 1.4 is that $\Gamma_{t}$ increases almost linearily with $n_{\text {sepx }}$ up to $n_{\text {sepx }} \approx 3-3.5$, after which $\Gamma_{t}$ begins to saturate. Following the definition of detachment in [3] for the ergodic divertor at Tore Supra: "departure from the high-recycling regime", this latter regime at $n_{\text {sepx }} \geq 3.5$ can be regarded as detachment.

\subsection{Spatially resolved recycling flux analysis}

We will now demonstrate that the behavior of the total recycling flux is not an artifact of the integration over the DED target. The calculated distribution of the particle flux to the DED target $\Gamma_{t \text {,local }}$ is shown in figure 5 (a) for a selected simulation with $n_{\text {sepx }}=1.0$ and $D_{\perp}=1.0$. It can be seen by a comparison to the magnetic footprint in figure 2 that particle flux arrives at the DED target both at the end of short magnetic flux tubes and long, chaotic magnetic field lines. The local particle flux density is extracted at several strike locations (A-D) and shown in figure $5(\mathrm{~b})$ and (c) for $D_{\perp}=0.2$ and $D_{\perp}=1.0$, respectively. It can be seen that, while the absolute value at distinct locations can be quite different, the general behavior with increasing $n_{\text {sepx }}$ remains the same: highrecycling-like behavior is found at each strike location in the $D_{\perp}=0.2$ case up to

$n_{\text {sepx }}=3.3$ after which simulations turn unstable, whereas in the $D_{\perp}=1.0$ case a saturation of $\Gamma_{t, \text { local }}$ at high densities is found at the same strike locations without a preceeding (strong) non-linear rise phase. 


\subsection{Remarks}

The supression of a high-recycling-like regime and the saturation of $\Gamma_{t}$ at high $n_{\text {sepx }}$ (i.e. detachment) in the high $D_{\perp}$ simulations might suggest that the friction between counter-flows due to cross-field momentum transfer is the underlying mechanism. This has been identified as the main mechanism for the absence of high-recycling at the W7AS stellarator [22]. However, we will show that this effect is - at least within the same model assumptions (and even same code) as used for W7-AS - not the dominant one for the TEXTOR helical divertor configuration.

\section{Interpretation of results}

For an interpretation of the simulation results, i.e. in order to identify the mechanism behind the absense of high-recycling and the saturation of the recycling flux at high $n_{\text {sepx }}$ for TEXTOR relevant $D_{\perp}$, we guide our analysis by the extended 2-point model [30] with correction factors $f_{\text {mom }}, f_{\text {cond }}, f_{\text {power }} \in[0,1]$, which relates upstream (index u) to target parameters (index t):

$$
\begin{array}{ll}
2 n_{t} T_{t} & =f_{\text {mom }} n_{u} T_{u} \\
T_{u}^{7 / 2} & =T_{t}^{7 / 2}+\frac{7}{2} \frac{f_{\text {cond }} q_{\|} L}{\kappa_{0 e}} \\
\left(1-f_{\text {power }}\right) q_{\|} & =q_{t}=\gamma n_{t} c_{s t} T_{t}
\end{array}
$$

The upstream density $n_{u}$ and heat flux $q_{\|}$are considered as control parameters, while the SOL length $L$, the sheath heat transmittion coefficient $\gamma=7$ and $\kappa_{0 e} \approx 2000$ are specified constants of the problem (The latter is a dimensionless constant for $T_{u}, T_{t}[\mathrm{eV}]$, $L[\mathrm{~m}]$ and $\left.q_{\|}\left[W \mathrm{~m}^{-2}\right]\right) . \quad c_{s t} \sim T_{t}^{1 / 2}$ is the sound speed at the target. The correction factors account for the presence of momentum losses (smaller $f_{\text {mom }}$ means larger losses), convective parallel heat transport (smaller $f_{\text {cond }}$ means larger contribution by convection, or any other heat transport channel other than parallel conduction) and volumetric power losses $\left(f_{\text {power }}\right.$ is the loss fraction of the total input power). According to this 2-point model, a mitigation of the high-recycling behavior or even a flux roll-over can be caused either by momentum losses $\left(\Gamma_{t} \sim f_{\text {mom }}^{2}\right)$ or by additional channels of heat transport $\left(\Gamma_{t} \sim f_{\text {cond }}^{4 / 7}\right)$. The former can be caused by direct losses from the plasma flow due to volume recombination. Other mechanisms to reduce $f_{\text {mom }}$ are charge exchange with recycling neutral particles, which is expected to drive the flux roll-over observed in poloidal divertor configurations, or cross-field particle diffusion and viscous transport acting on counter-flows, which has been concluded to be the relevant mechanism in stellarators like W7-AS [22] and the Tore Supra ergodic divertor [5]. The non-conductive heat flux corrections in $f_{\text {cond }}$ account for parallel heat convection along open field lines and, in particular in the TEXTOR helical divertor configuration, cross-field energy transport from the separatrix to the target. The direct impact of volumetric power losses is an increase of the recycling flux $\left(\Gamma_{t} \sim \frac{1}{1-f_{\text {power }}}\right)$, which is a consequence of a 
temperature reduction and the boundary condition for the target heat flux $q_{t}$ in $(7)$. The importance of these extensions of the original 2-point model is analysed in the subsequent sections.

\subsection{Momentum losses}

5.1.1. by charge exchange In order to find the relevant mechanism for the absenese of a high-recycling regime and the transition to detachment, we start with the investigation of momentum losses due to charge exchange with neutral particles. A second set of simulations with $D_{\perp}=1.0$ has been performed, but with the corresponding source term in the momentum balance (i.e the frictional force between neutral particles and ions) turned off in the code. The resulting recycling flux is shown in figure 4 by the blue dotted line. No significant change in $\Gamma_{t}$ is found compared to the reference case (blue solid line), which includes these momentum losses. Hence, charge exchange momentum losses are not responsible in our model for the observed absence of high-recycling like behavior and the saturation of $\Gamma_{t}$ in the TEXTOR helical divertor at high densities.

5.1.2. by volume recombination Volume recombination has not been activated in our present model. As can be seen in figure 8 (a), the temperature does not drop below a few eV, even in the high density cases. Hence, volume recombination is not relevant in the TEXTOR helical divertor. This is in agreement with experimental observations at TEXTOR [20] and also at the Tore Supra ergodic divertor [2, 5, 24].

5.1.3. by cross-field transport The impact of counter-flows at the DED target is estimated from the momentum balance (2). We consider the cross-field and parallel momentum fluxes

$$
\Gamma_{m \perp}=D_{\perp} \nabla_{\perp} m n u_{\|}, \quad \Gamma_{m \|}=m n u_{\|}^{2},
$$

and account for outward contribution of parallel transport along open perturbed field lines. The field line inclination due to RMPs in front of the DED target is $\theta=B_{\psi} / B \approx 0.008$ ( $B_{\psi}$ is the radial component of the magnetic field). We compare the two outward fluxes

$$
r_{\mathrm{mom}}=\frac{\Gamma_{m \perp}}{\theta \Gamma_{m \|}}
$$

and evaluate this ratio at the target $\left(u_{\|}=c_{s t}\right)$ for two counter-flows $\left(\nabla_{\perp} \approx 2 / \Delta x\right)$ :

$$
r_{\mathrm{mom}} \approx \frac{2 D_{\perp}}{\theta \Delta x c_{s t}}
$$

The poloidal distance can be extracted from figure 3 (f) to be $\Delta \vartheta \approx 20 \mathrm{deg}$, which translates to $\Delta x \approx 16 \mathrm{~cm}$ at $r \approx 47 \mathrm{~cm}$. This is already much larger than the radial extent of the perturbed plasma edge. 


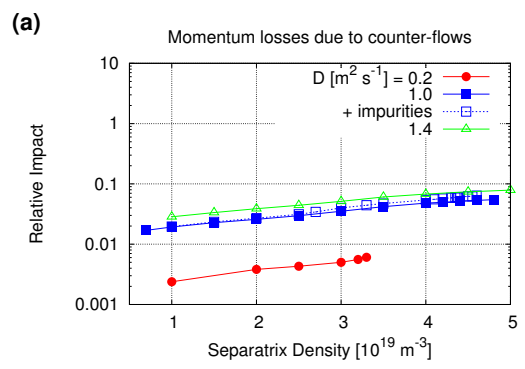

(b)

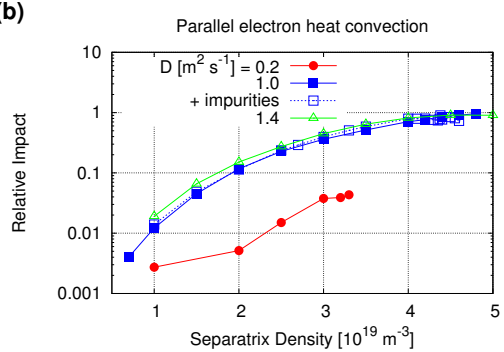

(c)

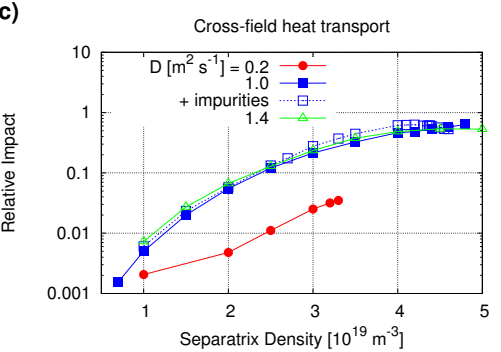

Figure 6. Impact factors for (a) cross-field momentum transport, (b) parallel electron heat convection and (c) cross-field heat transport.

(a)

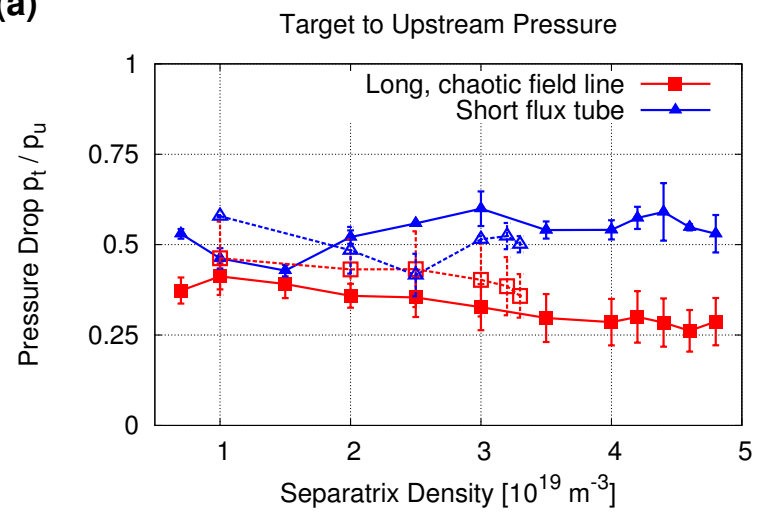

(b)

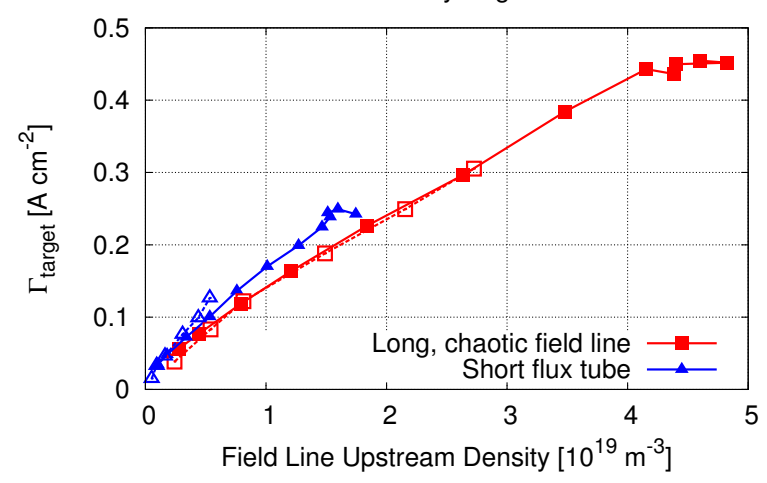

Figure 7. (a) Pressure drop $p_{t} / p_{u}$ along field lines and (b) local recycling flux for field line resolved upstream densities for simulations with $D_{\perp}=1.0$ (solid lines/filled symbols) and $D_{\perp}=0.2$ (dashed lines/open symbols). The short magnetic flux tube (blue, triangles) corresponds to strike location $\mathrm{C}$ in figure 5 and the long, chaotic field line (red, squares) to strike location D.

It can be seen in figure 6 (a) that the cross-field viscosity acting in the counter-flows is of minor importance (i.e. $r_{\text {mom }}<10 \%$ ). A similar analysis for typical parameters of the W7-AS stellarator leads to the opposite conclusion [22], because $\theta \cdot \Delta x$ is by a factor of 30 smaller for the W7-AS configuration. Note that at Tore Supra this effect might indeed be relevant, as it has been concluded in [5], because of the much higher mode number of the flow pattern $(m=18)$ [36] and, consequently, the shorter poloidal distance between counter-flows.

A more accurate analysis can be given for selected field lines. We consider the 2 -point momentum balance (5) and calculate the pressure drop $r_{\mathrm{P}}=p_{t} / p_{u}$. This ratio is shown in figure 7 (a) for two selected field lines: a short field line within a magnetic flux tube (blue), and a long, chaotic field line (red). $r_{\mathrm{P}}$ is averaged over the two target strike points of each field line. As there are several stagnation points (i.e. upstream locations) along the long, chaotic field line, the pressure drop is calculated between the last stagnation point and the target. 
(a)

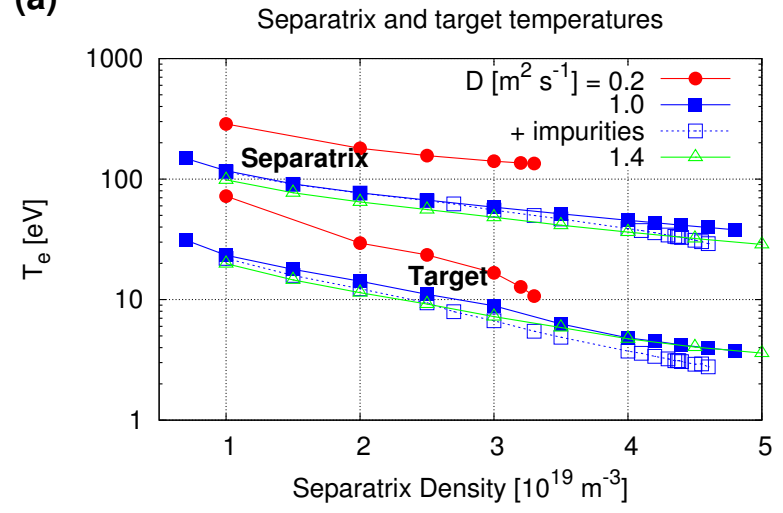

(b)

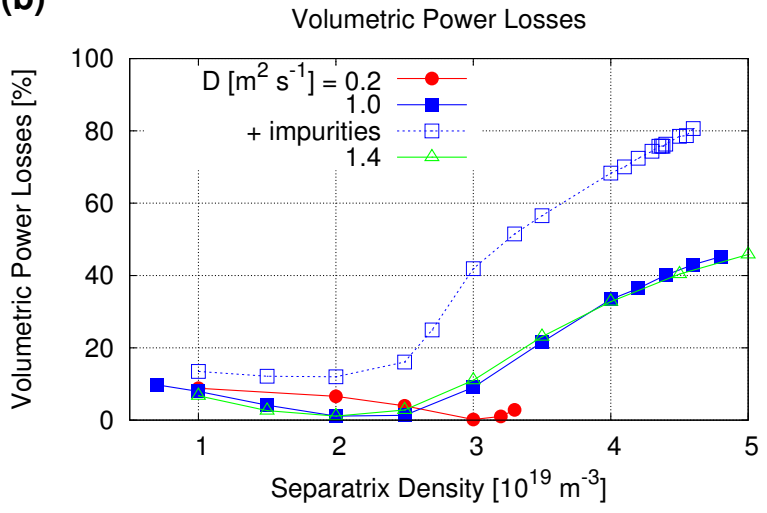

Figure 8. (a) Separatrix and target temperatures for electrons $T_{e s}$ and $T_{e t}$, respectively (the ion temperature behaves in the same way) and (b) volumetric power losses (relative to $P_{\text {in }}$ ) due to radiation and ionization of hydrogen and impurities.

It is shown in figure 7 (a) that $r_{\mathrm{P}}$ stays at a level of 0.5 in case of the short magnetic flux tube, which is the expected value of the 2-point model without momentum losses. Nevertheless, a weak reduction of $r_{\mathrm{P}}$ from $\sim 0.4$ to $\sim 0.3$ at high densities is found for the long, chaotic field line. Allthough this might suggest that momentum losses in the chaotic domain do have some relevance, a significant increase in momentum loss at the transition to detachment is not observed in the simulations. In particular the fact that absence of strong high-recycling and a transition to detachment is observed at all strike locations (as shown in figure 5 (c)) suggests that momentum losses are not the driving mechanism behind the observed recycling flux behavior at TEXTOR. Furthermore, weak momentum losses are also present in the $D_{\perp}=0.2$ simulations (red dashed line) despite strong high-recycling behavior in this case.

Finally, a closer analysis of the recycling behavior at selected strike points reveals that the non-linear increase of $\Gamma_{t \text {,local }}$ for the $D_{\perp}=0.2$ case in figure 5 (b) is related to the non-linear increase of the upstream density $n_{u, \mathrm{fl}}$ of the corresponding field lines. The upstream density for the two examplary field lines from above has been extracted from the 3D simulation results and is used in figure 7 (b) as the "control parameter". This field line resolved analysis shows that linear recycling behavior is present also in the $D_{\perp}=0.2$ case (in which friction by counter-flows is naturally mitigated), and hence, that the early detachment in the $D_{\perp}=1.0$ and 1.4 cases must be caused by some other mechanism.

\subsection{Temperature effects}

Combining the 2-point momentum balance (5) and the heat flux boundary condition (7) gives

$$
\Gamma_{t} \sim \frac{\left(f_{\mathrm{mom}} n_{u} T_{u}\right)^{2}}{q_{\|}\left(1-f_{\text {power }}\right)},
$$


which indicates that a reduction of upstream/separatrix temperature by additional heat transport channels must be the relevant mechanism. It can be seen in figure 8 (a) that the upstream/separatrix temperature indeed drops with increasing $n_{\text {sepx }}$. For $D_{\perp}=1.0$ we find that $T_{u}$ drops from $110 \mathrm{eV}$ to $40 \mathrm{eV}$ when $n_{\text {sepx }}$ is increased from 1 to 5 and volumetric power losses increase from $f_{\text {power }}=0$ to 0.4 (see figure $8(\mathrm{~b})$ ). We apply $f_{\text {mom }} \approx 1$ and find that $\Gamma_{t}$ must increase by a factor of 6 according to (11), which can indeed found in the simulations in figure 4.

5.2.1. Additional heat transport channels We now support the relevance of additional outward heat flux channels by a rough estimate of the impact of parallel electron heat convection at the DED target and cross-field heat transport. We consider the ratios

$$
r_{\mathrm{conv}}=\frac{q_{\| \mathrm{conv}}}{q_{\| \mathrm{cond}}}=\frac{\frac{5}{2} n T_{e} u_{\|}}{\kappa_{e} \nabla_{\|} T_{e}}
$$

and

$$
r_{\text {cross }}=\frac{q_{\perp}}{\theta q_{\| \text {cond }}}=\frac{\frac{5}{2} T_{e} D_{\perp} \nabla_{\perp} n+n \chi_{e} \nabla_{\perp} T_{e}}{\theta \kappa_{e} \nabla_{\|} T_{e}},
$$

where $\theta$ in (13) again accounts for the radial contribution of parallel transport along open perturbed field lines. We "post-process" the simulation results and average $q \|$ conv and $q_{\| \text {cond }}$ in front of the DED target (on the last 0.3 poloidal turns of a field line). We estimate the cross-field gradients in (13) to

$$
\nabla_{\perp} n \approx \frac{n_{\mathrm{sepx}}-n_{t}}{\Delta r_{\mathrm{edge}}}, \quad \nabla_{\perp} T_{e} \approx \frac{T_{e, \mathrm{sepx}}-T_{e t}}{\Delta r_{\mathrm{edge}}}
$$

where $\Delta r_{\text {edge }} \approx 12 \mathrm{~cm}$ is the radial extend of the perturbed edge layer. It can be seen in figures 6 (b) and (c) that both heat transport channels become more important with increasing $n_{\text {sepx }}$ and are dominant for high $n_{\text {sepx }}$ in the $D_{\perp}=1.0-1.4$ cases. These findings are consistent with those from an improved 2-point model for the limiter SOL at TEXTOR [31], which also show that convective heat transport remains significant at high densities. The origin of strong convective parallel heat fluxes is a high fraction of upstream particle sources, which are of the order $50 \%$ of the total recycling flux.

The low relevance of additional heat transport for $D_{\perp}=0.2$ is consistent with the global high-recycling-like behavior in this case (although this global analysis doesn't resolve the fact that the field line upstream density $n_{u, \mathrm{fl}}$ increases non-linearily with $n_{\text {sepx }}$ rather than $\Gamma_{t \text {,local }}$ with $\left.n_{u, \mathrm{fl}}\right)$. The relative high temperatures in this case explain the low values of $r_{\text {conv }}$ and $r_{\text {cross }}\left(\kappa_{e} \sim T_{e}^{5 / 2}\right)$.

The presence of a high-recycling regime at Tore-Supra might be related to the higher input powers $(1-6.5 \mathrm{MW})$ [23]. Edge temperatures in this regime decrease from 40 to $15 \mathrm{eV}$, which is consistent with the values shwon in figure 8 (a) for the high-recycling case $D_{\perp}=0.2$. The access to the high-recyling regime at Tore-Supra is given by the condition $[23]$

$$
P_{\text {in }}[\mathrm{MW}] \gtrsim 4.15 \cdot 10^{-2}\left\langle n_{e}\right\rangle^{3.4},
$$



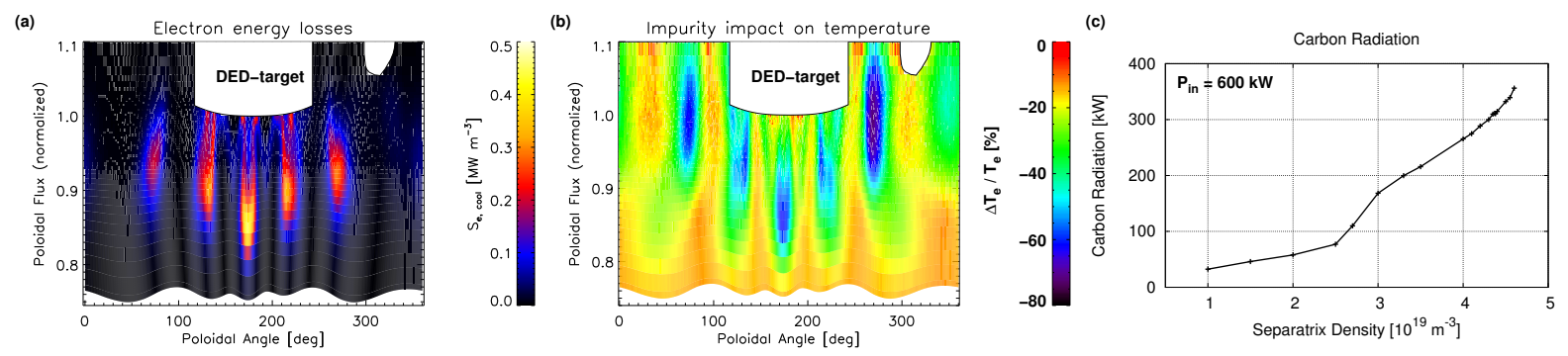

Figure 9. (a) Power losses due to carbon radiation and ionization and (b) the corresponding relative reduction of electron temperature $\Delta T_{e} / T_{e}$ at $n_{\mathrm{sepx}}=4.0$ as $2 \mathrm{D}$ cuts at $\varphi=0$ deg. (c) Total power losses due to the presence of impurities.

where $\left\langle n_{e}\right\rangle$ is the volume averaged density. Such a scaling law indicates that a direct transition from the linear to the detached regime might occur before a high-recycling regime can be established, if the input power is low enough.

5.2.2. Impact of impurities Guided by the observation in figure 8 (b) that volumetric power losses are present in the early detachment cases $D_{\perp}=1.0-1.4$, but not in the highrecycling-like case $D_{\perp}=0.2$, we now investigate the impact of additional volumetric power losses by impurity radiation. Note that $f_{\text {power }}$ in the 2-point model (5)-(7) only considers downstream power losses. Upstream power losses require a modification of (6) and lead to a reduction of upstream temperature.

Carbon impurities are naturally released when the plasma comes in contact with the graphite plates at the DED target. Production and transport of such impurities is taken into account during a dedicated density scan for $D_{\perp}=1.0$. Their impact on the edge plasma is taken into account by an additional cooling term $S_{e, \text { cool }}$ for electrons, reflecting energy losses due to impurity ionization and radiation. Impurity sources are approximated by a sputter coefficient $c_{\text {sput }}=2 \%$ applied to the incident hydrogen ion flux. The following analysis is focused on the resulting impact on the recycling flux $\Gamma_{t}$, while an analysis of the modification of impurity sources and core contamination (as has been done for Tore Supra, see e.g. $[4,37])$ is beyond the scope of this article.

A $2 \mathrm{D}$ cut of the impurity related power losses for $n_{\text {sepx }}=4.0$ is shown in figure 9 (a). Significant, localized power losses occur in the plasma edge between $\Psi_{n} \approx 0.8$ and 1.0. The corresponding impact on the electron temperature is shown in figure $9(\mathrm{~b})$. A strong impact on $T_{e}$ is found at poloidal locations with large power losses, at which $T_{e}$ is reduced by more than $60 \%$. A weaker impact is found at the inner simulation boundary $(-15 \%)$ and at the DED target $(-22 \%)$. The total power losses due to impurities are shown in figure 9 (c), demonstrating that in our model at $n_{\text {sepx }}=4.0$ already more than $40 \%$ of the heating power $\left(P_{\text {in }}=600 \mathrm{~kW}\right)$ is lost due to radiation from intrinsic carbon impurities.

As can be seen by the blue dashed profile in figure 4, the presence of carbon impurities results in a strong reduction of recycling flux for $n_{\text {sepx }} \geq 3.0$ (i.e. compared to 
the reference case, blue solid line). A peak value of $\Gamma_{t}=7.2 \mathrm{kA}$ is reached at $n_{\mathrm{sepx}}=3.5$, which is a reduction of $25 \%$ in $\Gamma_{t}$ with respect to the corresponding simulation without impurities. The recycling flux then drops to $\Gamma_{t}=5.7 \mathrm{kA}$ at $n_{\mathrm{sepx}}=4.6$, which is by a factor of 2 smaller than the reference case.

The strong modification of $\Gamma_{t}$ compared to the reference case is coincident with a strong increase of power losses by impurities (compare figures 4 and 9 (c)). Such a strong increase just before the transition to detachment is also found at Tore Supra [37], and is essentially a recution of the available heating power. A significant amount of power losses occur at upstream positions of individual field lines, at which the temperature is reduced by at least $40 \%$. This is additional evidence that temperature effects are responsible for the absence of high-recycling behavior and the early transition to detachment in the TEXTOR helical divertor.

\section{Conclusions}

The TEXTOR helical divertor in $m / n=6 / 2$ configuration has been studied by 3D computer simulations. It has been shown that no high-recycling regime is established, but rather an early transition to a detached state (characterized by a saturation or even roll-over of the recycling flux at high densities). This findings are in agreement with experimental observations for configurations with the same poloidal/toroidal base mode number.

The driving mechansim for this behavior in the EMC3-EIRENE model has been identified as a reduction of upstream temperature rather than momentum losses. The former is caused by increased outward heat transport due to parallel heat convection along open, perturbed magnetic field lines and cross-field heat transport from the separatrix to the target. Furthermore, upstream power losses, e.g. by carbon impurities, result in a reduction of upstream temperature as well. This analysis strongly indicates that the relative importance of recycling and transport mechanisms in the TEXTOR helical divertor (i.e. dominant temperature effects) is significantly different from that in other divertor machines where momentum losses, in the right proportion to power losses, are thought to play a key role for transition to the detached divertor state.

\section{References}

[1] Ghendrih P, et al., 1999 J. Nucl. Mater. 266-269 189. doi:10.1016/S0022-3115(98)00525-X

[2] Meslin B, et al., 1999 J. Nucl. Mater. 266-269 318. doi:10.1016/S0022-3115(98)00875-7

[3] Ghendrih P, et al., 2001 Nuclear Fusion 41 1401. doi:10.1088/0029-5515/41/10/308

[4] Ghendrih P, et al., 2002 Nuclear Fusion 4210 1221. doi:10.1088/0029-5515/42/10/308

[5] Ghendrih P et al., 2009 Fusion Science and Technology $\mathbf{5 6} 1432$

[6] Finken K, et al., 1998 Nuclear Fusion 38 515. doi:10.1088/0029-5515/38/4/304

[7] Finken K, et al., 1999 Nuclear Fusion 395637

[8] Abdullaev S, et al., 2001 Phys. Plasmas 862739

[9] Masuzaki S, et al., 2002 Nuclear Fusion 42 750. doi:10.1088/0029-5515/42/6/313 
[10] Kobayashi M, et al., 2007 Journal of Nuclear Materials 363-365 $294 . \quad$ doi: 10.1016/j.jnucmat.2007.01.038

[11] Jakubowski M, et al., 2004 Nuclear Fusion 446 S1. doi:10.1088/0029-5515/44/6/S01

[12] Lehnen M et al., 2005 Plasma Phys. Control. Fusion 47 B237. doi:10.1088/0741-3335/47/12B/S18

[13] Jakubowski M, et al., 2006 Phys. Rev. Lett. 96 035004. doi:10.1103/PhysRevLett.96.035004

[14] Schmitz O, et al., 2007 Journal of Nuclear Materials 363-365 $680 . \quad$ doi: 10.1016/j.jnucmat.2007.01.067

[15] Schmitz O, et al., 2008 Nuclear Fusion 48 024009. doi:10.1088/0029-5515/48/2/024009

[16] Evans T E, et al., 2006 Nature Physics 2 419. doi:10.1038/nphys312

[17] Liang Y, et al., 2007 Phys. Rev. Lett. 98265004 1. doi:10.1103/PhysRevLett.98.265004

[18] Loarte A, et al., 2002 Plasma Phys. Control. Fusion 44 1815. doi:10.1088/0741-3335/44/9/303

[19] Federici G, et al., 2003 Journal of Nuclear Materials 313-316 11. doi:10.1016/S0022$3115(02) 01327-2$

[20] Clever M, 2010. Hydrogen recycling and transport in the helical divertor of TEXTOR. Schriften des Forschungszentrum Jülich, Reihe Energie \& Umwelt, Band 87. ISBN 978-3-89336-673-6

[21] Clever M, et al., 2011 Nuclear Fusion "Experimental investigation of density regimes in the helical divertor at TEXTOR" (submitted to)

[22] Feng Y, et al., 2006 Nuclear Fusion 46 807. doi:10.1088/0029-5515/46/8/006

[23] Azéroual A, et al., 2000 Nuclear Fusion 40 1651. doi:10.1088/0029-5515/40/9/309

[24] Loarer T et al., 2009 Fusion Science and Technology $\mathbf{5 6} 1300$

[25] Feng Y, et al., 1999 Journal of Nuclear Materials 266-269 812. doi:10.1016/S0022-3115(98)008447

[26] Feng Y, et al., 2004 Contrib. Plasma Phys. 44 1-3 57. doi:10.1002/ctpp.200410009

[27] Reiter D, et al., 2005 Fusion Science and Technology 472172

[28] Kobayashi M, et al., 2004 Contrib. Plasma Phys. 44 1-3 25. doi:10.1002/ctpp.200410003

[29] Kobayashi M, et al., 2004 Nuclear Fusion 44 S64. doi:10.1088/0029-5515/44/6/S07

[30] Stangeby P, 2000 The Plasma Boundary of Magnetic Fusion Devices. Institute of Physics Publishing Bristol and Philadelphia

[31] Tokar M Z, et al., 2004 Phys. Plasmas 11 4610. doi:10.1063/1.1791232

[32] Kotov V et al., 2009 Plasma Phys. Control. Fusion 51 115002. doi:10.1088/0741$3335 / 51 / 11 / 115002$

[33] Braginskii S, 1965 Review of Plasma Physics 1205

[34] Lehnen M, et al., 2005 J. Nucl. Mater. 337-339 171. doi:10.1016/j.jnucmat.2004.10.100

[35] Lehnen M, et al., 2008 Plasma and Fusion Research 3 S1039. doi:10.1585/pfr.3.S1039

[36] Gunn J P, et al., 2001 J. Nucl. Mater. 290-293 877. doi:10.1016/S0022-3115(00)00468-2

[37] Corre Y, et al., 2007 Nuclear Fusion 47119 doi:10.1088/0029-5515/47/2/006 